Proceedings of the International School and Conference on Optics and Optical Materials, ISCOM07, Belgrade, Serbia, September 3-7, 2007

\title{
Transmission Spectra of Thue-Morse Multilayers Containing Negative Index Metamaterials
}

\author{
M. MAKSIMOVIC ${ }^{a, *}$ AND Z. JAKSIC ${ }^{b}$ \\ ${ }^{a}$ MESA + Institute for Nanotechnology, University of Twente, The Netherlands \\ ${ }^{b}$ Institute of Microelectronic Technologies and Single Crystals \\ University of Belgrade, Serbia
}

\begin{abstract}
We study the optical transmission spectra in one-dimensional aperiodic Thue-Morse multilayers composed from alternating layers of media with positive and negative refractive index. We examine the influence of phase compensation on the spectral transmission for both on-axis and off-axis wave propagation. The origin of the transmission resonances and their relation with the field localization are analyzed. Nondispersive and lossless, as well as realistic dispersive and weakly lossy materials are considered.
\end{abstract}

PACS numbers: 42.70.Qs, 71.36.+c, 71.55.Jv, 78.20.Ci

\section{Introduction}

Negative refractive index metamaterials (NIM) are artificially structured media with sub-wavelength features and with simultaneously negative dielectric permittivity and magnetic permeability [1-3]. The direction of the Poynting vector $\boldsymbol{S}$ in NIM is opposite to that of the wave vector $\boldsymbol{k}$, i.e. electric, magnetic field and wave vectors $\{\boldsymbol{E}, \boldsymbol{H}, \boldsymbol{k}\}$ form a left-oriented triplet. This is the reason why such media were dubbed "left-handed materials", contrary to the conventional "right-handed materials" (positive refractive index, PIM). Large efforts have been dedicated to the NIM research in the recent years ([1-3] and references therein). The operating wavelengths have been progressively decreasing and negative refractive behavior has been observed in the optical range [4].

Multilayers with NIM part and with periodic [5, 6], quasi-periodic (Fibonnacci-type) [7], or pre-fractal (Cantor-type) [8, 9] layer arrangements, attracted attention due to their many peculiar properties with both theoretical and practical interest.

*corresponding author; e-mail: m.maksimovic@math.utwente.nl 
We investigate non-periodic one-dimensional stacks composed of alternating layers of media with positive and negative refractive index. The stacks follow a design rule based on the model aperiodic Thue-Morse ( $\mathrm{T}-\mathrm{M})$ substitution sequence [10-14]. All-PIM T-M type multilayers possess interesting and useful spectral properties, such as self-similarity, spectral scalability and well-localized multiple resonances in their optical transmission spectra [10-14]. We employ the standard transfer matrix method for numerical computations and analysis [15]. Some results on T-M multilayers with NIM are obtained in $[16,17]$ with the prediction of a new type of a non-Bragg, zero- $n$ bandgap (where the averaged value of the refractive index in the structure is close to zero).

We dedicate our attention to the resonances in the transmission spectra and the field distributions associated with them for finite structures with both on-axis and off-axis wave propagation. The angular dependence of the transmission spectra and of the resonances robustness with respect to the phase shift modulation are investigated. Transmission spectra of $\mathrm{T}-\mathrm{M}$ multilayer with dispersive and lossy NIM parts are considered.

\section{Thue-Morse multilayers containing NIM}

A Thue-Morse substitution sequence can be defined via a binary alphabet $\{\mathrm{A}, \mathrm{B}\}$ and a set of simple mapping rules $\mathrm{A} \rightarrow \mathrm{AB}, \mathrm{B} \rightarrow \mathrm{BA}$, with the symbol $\mathrm{A}$ as an initiator. The first several T-M generations are A, AB, ABBA, ABBABAAB, ABBABAABBAABABBA, etc. A schematic presentation of T-M multilayers and of a single NIM-PIM interface is shown in Fig. 1.

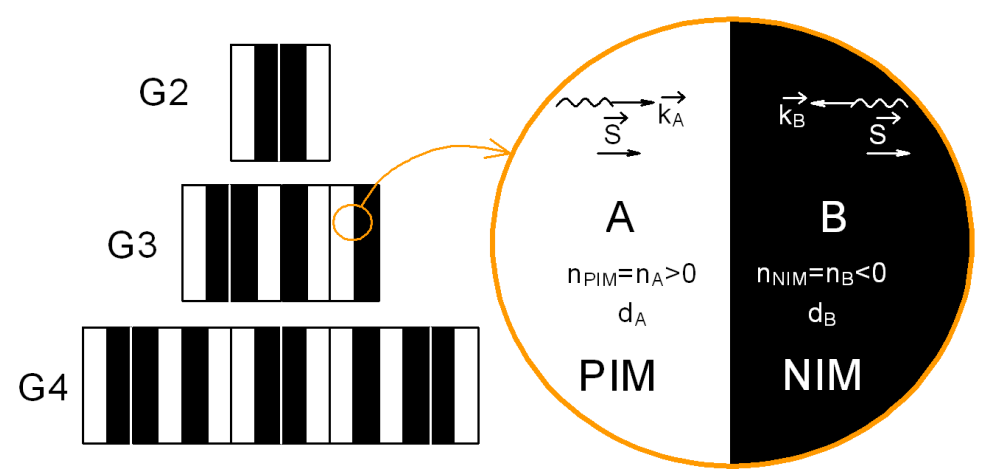

Fig. 1. Left: aperiodic structures composed of NIM and PIM strata arranged as ThueMorse sets for second, third and fourth generation; right: schematic presentation of a negative index-positive index material interface.

Even-order T-M multilayers possess a mirror symmetry in refractive index distribution. The number of elements in the sequence increases in the $n$-th generation as $2^{n}$, while the number of different elements $\mathrm{A}$ and $\mathrm{B}$ remains equal. We take two refractive indices $\left(n_{\mathrm{A}}, n_{\mathrm{B}}\right)$ and physical lengths $\left(d_{\mathrm{A}}, d_{\mathrm{B}}\right)$ that correspond 
to $(\mathrm{A})$ and $(\mathrm{B})$ in the $\mathrm{T}-\mathrm{M}$ sequence. The refractive index in the incident and in the output medium is assumed to be equal $\left(n_{0}=1\right)$.

First, we chose frequency independent values of refractive indices $n_{\mathrm{A}}=1.41$ $\left(\mu_{\mathrm{A}}=1, \epsilon_{\mathrm{A}}=2\right), n_{\mathrm{B}}=-2\left(\mu_{\mathrm{B}}=-1, \epsilon_{\mathrm{B}}=-4\right)$, for the purpose of comparison with the prior results in literature without a loss of generality. We introduce the reduced optical thickness $n_{\mathrm{A}} d_{\mathrm{A}}=\alpha \lambda_{0}, n_{\mathrm{B}} d_{\mathrm{B}}=\beta \lambda_{0}$, with the phase shifts in the corresponding layers given as $\delta_{\mathrm{A}}=2 \pi \alpha \Omega \cos \left(\theta_{\mathrm{A}}\right)$ and $\delta_{\mathrm{B}}=2 \pi \beta \Omega \cos \left(\theta_{\mathrm{B}}\right)$, where $\Omega=\lambda_{0} / \lambda=\omega / \omega_{0}$ is the normalized frequency/wavelength with $\omega_{0}$ and $\lambda_{0}$ being reference frequency and wavelength.

In the case of oblique incidence the angles of propagation $\theta_{\mathrm{A}}, \theta_{\mathrm{B}}$ are obtained via Snell's law $n_{0} \sin \left(\theta_{0}\right)=n_{\mathrm{A}} \sin \left(\theta_{\mathrm{A}}\right)=\mathrm{n}_{\mathrm{B}} \sin \left(\theta_{\mathrm{B}}\right)$, where $\theta_{0}$ is the incidence angle in the input medium. When $\alpha=\beta=0.25$ we are dealing with a quarter-wavelength optical thickness, which is the usual choice in literature.

The interplay between the order and the phase compensation (partial or full removal of the phase shift after propagating through a PIM-NIM structure) greatly affects the spectral resonances in the stacks based on different ordering schemes. Periodic multilayers with NIM exhibit wide band gaps and flattened transmission [13-16], while T-M multilayers exhibit a similar behavior with the distinctive feature of the existence of resonances in the middle of the Bragg band gap, as seen in Fig. 2a. Multiple resonances in the spectra of all-PIM periodic or $\mathrm{T}-\mathrm{M}$ multilayers are not present in the PIM-NIM structures due to phase compensation, Fig. 2b, c. While the quasi-periodic and pre-fractal Cantor NIM-containing multilayers possess self-similar and scalable spectra for higher generations $[4,5]$, in $\mathrm{T}-\mathrm{M}$ multilayers these spectral properties are absent, even in the dispersionless and lossless case, see Fig. $2 \mathrm{~b}$ and c.

In PIM-NIM T-M multilayers only a midgap resonance at $\Omega=1$ is present for normal incidence, due to a special spatial correlation in the $\mathrm{T}-\mathrm{M}$ multilayer. The intensity field distribution is identical for both all-PIM and PIM-NIM structures and follows the structure of the T-M sequence, as shown in Fig. 2d, e. Alternating symmetric and asymmetric arrangements of layers in successive generations of the T-M multilayers determines the nature of the spectral resonances, i.e. perfect transmission exists in mirror symmetric structures even for oblique incidence. In asymmetric multilayers a perfect transmission resonance at $\Omega=1$ exists only for normal incidence and the quarter-wavelength optical thickness (equal phase shifts).

The resonance shift and the pronounced angular dependence, leading to the appearance of multiple resonances for oblique incidence, as shown in Fig. 3a, b, is in contrast with some predictions that the transmission spectra of PIM-NIM structures are generally less sensitive to the incidence angle [13-15]. The number and the positions of transmission resonances are very sensitive to the modulation of the optical thickness $[9,10]$ which is clearly seen in Fig. 3c for a 5th generation $\mathrm{T}-\mathrm{M}$ multilayer (higher generations show similar behavior). 

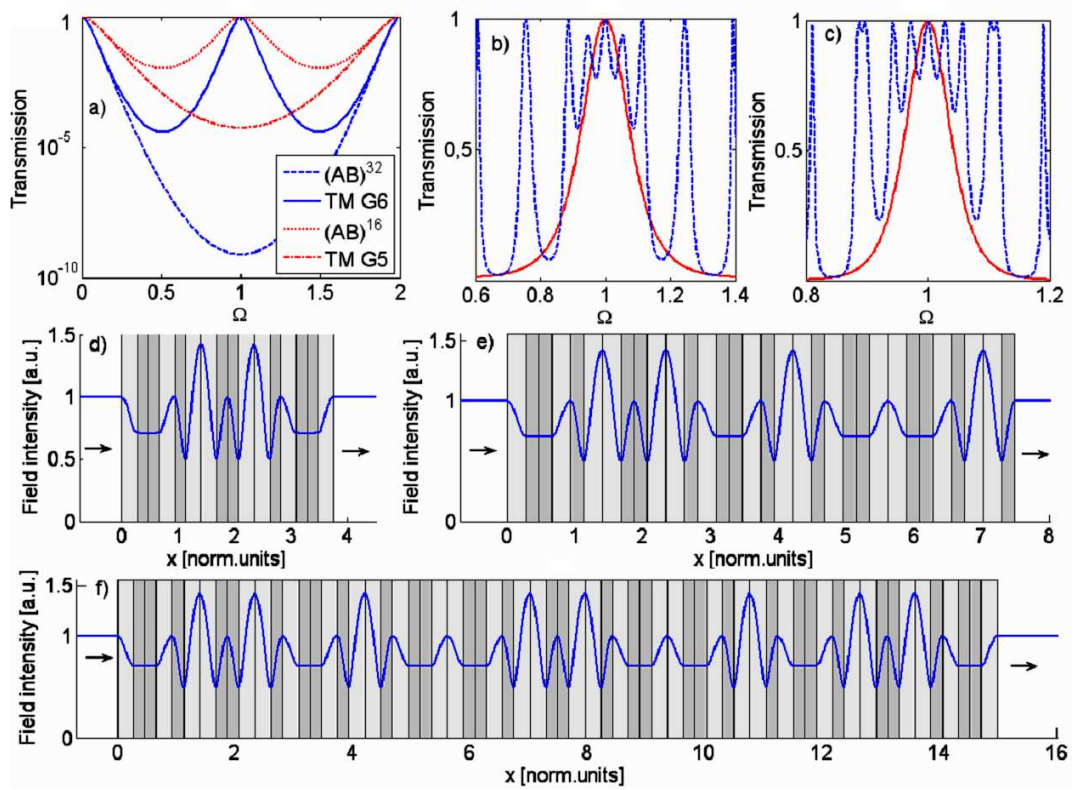

Fig. 2. Direct comparison of transmission spectra for periodic structures and $\mathrm{T}-\mathrm{M}$ multilayers (solid) with an equal number of layers in the stack (a) and equal quarter-wavelength optical thickness. Transmission spectra for 5th generation (b) and 6th generation (c) T-M multilayer with all-PIM (dash) and NIM-PIM (solid). Intensity profile for normal incidence in all-PIM and PIM-NIM T-M multilayer for 4th (d), 5th (e) and 6th generation (f) and $\Omega=1$.

All NIM realized until now are dispersive and lossy, thus finally we choose more realistic and very strong Drude-type dispersion relations

$$
\varepsilon(\omega)=1-\frac{\omega_{\mathrm{pe}}^{2}}{\omega\left(\omega-\mathrm{i} \Gamma_{\mathrm{e}}\right)}, \quad \mu(\omega)=1-\frac{\omega_{\mathrm{pm}}^{2}}{\omega\left(\omega-\mathrm{i} \Gamma_{\mathrm{m}}\right)},
$$

where $\omega_{\mathrm{pe}(\mathrm{m})}$ are the electric (magnetic) artificial plasma frequencies and $\Gamma_{\mathrm{e}(\mathrm{m})}$ electric (magnetic) damping constants (which can be expressed as a fraction of the plasma frequency $[1-5,9])$. We chose $\omega_{\mathrm{pe}}=\omega_{\mathrm{pm}}=\omega_{\mathrm{p}}$ and $\Gamma_{\mathrm{e}}=\Gamma_{\mathrm{m}}=\Gamma=\omega_{\mathrm{p}} 10^{-6}$, thus the refractive index of the NIM part reads

$$
n(\omega)=\sqrt{\varepsilon(\omega) \mu(\omega)} \approx 1-\frac{\omega_{\mathrm{p}}^{2}}{\omega^{2}}-\mathrm{i} 10^{-6} \quad \text { and } \quad n(\Omega) \approx 1-\frac{\Omega_{\mathrm{p}}^{2}}{\Omega^{2}}-\mathrm{i} 10^{-6} .
$$

We assumed very small but still present losses, thus avoiding an electromagnetic nihility (material parameters locally equal to zero). Parameters for the NIM part are chosen in a such way that the physical thickness is given according to the condition $\left|n_{\mathrm{B}}\right| d_{\mathrm{B}}=0.25 \lambda_{0}$ for $n_{\mathrm{B}}(\Omega=1)=-2$ (and $\Omega_{\mathrm{p}}^{2}=3$ ), while the parameters in the PIM part are the same as before. In this way both modulations of the 

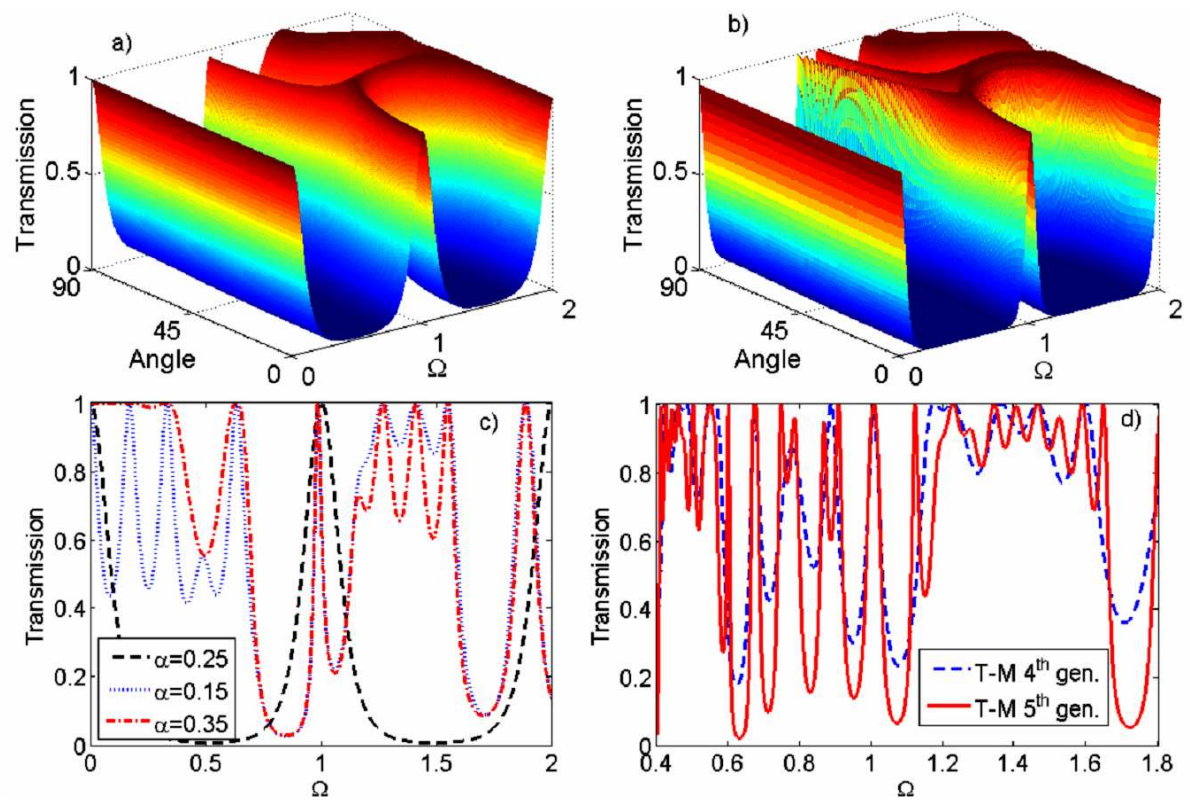

Fig. 3. Transmission spectra for oblique incidence: the 5 th generation (a) and the 6 th generation T-M multilayer (b). Transmission spectra for varying layer thickness parameter $\alpha$ (with $\alpha+\beta=0.5$ ) at normal incidence and 5th T-M multilayer (c). Transmission spectra for normal incidence and with (strong) Drude-type frequency dispersion in the NIM part and small loss (d).

scattering strength (refractive index contrast) and the phase shift are present [9]. Transmission spectra with many resonances appear (Fig. 3d), but without obvious special spectral properties arising from the order or the material properties.

\section{Conclusions}

We have presented transmission spectra for the Thue-Morse multilayers composed from alternating layers with positive and negative refractive indices. In contrast to other non-periodic NIM-containing multilayers where self-similar and scalable spectra occurred for higher generations, for T-M multilayers these spectral properties do not appear, even in the dispersionless and lossless case. While the periodic structures with NIM exhibit wide band gaps, T-M multilayers exhibit transmission resonances located at the midgap frequency of the periodic multilayer. The field distribution at the resonance frequency suggests a common origin in both all-PIM and NIM-containing multilayers connected with the existence of special spatial correlation irrespective of internal mirror symmetry. The position of this resonance is very sensitive to the incidence angle and the phase-shift modulation, suggesting that the phase compensation is most effective in equal-phase shift structures. The introduction of realistic material dispersion in the NIM material 
introduces both phase shift and scattering strength modulation in the structure. This increases the number of transmission resonances even under normal incidence conditions and effectively diminishes the influence of the aperiodic order and the phase compensation. The prospect of a weak material dispersion in the NIM leads to a potential for the application of the optical phenomena associated with aperiodic order.

\section{References}

[1] S.A. Ramakrishna, Rep. Prog. Phys. 68, 449 (2005).

[2] Z. Jaksic, N. Dalarsson, M. Maksimovic, Microwave Rev. 12, 1 (2006).

[3] K. Busch, G. von Freymann, S. Linden, S. Mingaleev, L. Tkeshelashvili, M. Wegener, Phys. Rep. 444, 101 (2007).

[4] C.M. Soukoulis, S. Linden, M. Wegener, Science 315, 47 (2007).

[5] I.S. Nefedov, S.A. Tretyakov, Phys. Rev. E 66, 036611 (2002).

[6] J. Li, L. Zhou, C.T. Chan, P. Sheng, Phys. Rev. Lett. 90, 083901 (2003).

[7] J. Li, D. Zhao, Zh. Liu, Phys. Lett. A 461, 332 (2004).

[8] J. Gerardin, A. Lakhtakia, Phys. Lett. A 301, 377 (2002).

[9] M. Maksimovic, Z. Jaksic, J. Opt. A 8, 355 (2006).

[10] R. Merlin, IEEE J. Quantum Electron. 24, 1791 (1988).

[11] E. L. Albuquerque, M.G. Cottam, Phys. Rep. 376, 225 (2003).

[12] E. Macia, Rep. Prog. Phys. 69, 397 (2006).

[13] N.H. Liu, Phys. Rev. B 55, 3543 (1997).

[14] H. Lei, J. Chen, G. Nouet, S. Feng, Q. Gong, X. Jiang, Phys. Rev. B 75, 205109 (2007).

[15] J.A. Kong, Prog. Electromag. Research 35, 1 (2002).

[16] J. Monsoriu, R. Depine, E. Silvestre, J. Eur. Opt. Soc. 2, 07002 (2007).

[17] F.F. de Medeiros, E.L. Albuquerque, M.S. Vasconcelos, J. Phys. C, Condens. Matter 14, 8737 (2006). 